\title{
Axial-vector mesons in a relativistic point-form approach
}

\author{
A. Krassnigg \\ Physics Division, Argonne National Laboratory, Argonne, IL 60439, USA
}

(Dated: November 5, 2018)

\begin{abstract}
The Poincaré invariant coupled-channel formalism for two-particle systems interacting via oneparticle exchange, which has been developed and applied to vector mesons in Ref. [1] is applied to axial vector mesons. We thereby extend the previous study of a dynamical treatment of the Goldstone-boson exchange by comparison with the commonly used instantaneous approximation to the case of orbital angular momentum $l=1$. Effects in the mass shifts show more variations than for the vector-meson case. Results for the decay widths are sizable, but comparison with sparse experimental data is inconclusive.
\end{abstract}

PACS numbers: 12.39.Ki,21.45.+v

\section{INTRODUCTION}

During the past years, constituent quark models have been used successfully to calculate spectra and properties of hadrons. In the course of the developement of these models, relativity has emerged as a key ingredient in the light-quark sector. This has found early consideration; e.g. in the well-known works of Godfrey and Isgur 2] and Capstick and Isgur 3], where relativistic corrections were introduced in a nonrelativistic potential model; Feynman et al. 4] based their model on a relativistic harmonic oscillator; Carlson et al. [5] used relativistic kinetic energies plus flux-tube motivated potential terms. In the particular case of the Goldstone-bosonexchange (GBE) constituent-quark model (CQM) 6] the early nonrelativistic formulation [7, 8] showed severe inconsistencies and was soon superseded by a "semirelativistic" version [9]. In the semirelativistic treatment the Hamiltonian of the model contains the relativistic kinetic energy plus potential terms, and it can in fact be reinterpreted as the mass operator of a Poincaré invariant model as long as the potential terms are rotationally invariant and do not depend on the total momentum of the bound state [10]. In this form the GBE CQM has been applied to spectroscopy of the light and strange baryon sector with wide success; however, an analogous calculation for mesons seemed to indicate failure of the model in this sector 11]. Only recently [1] a Poincaré invariant coupled-channel (CC) formalism for confined two-particle systems interacting via one-particle exchange has been shown to elucidate the relevance and applicability of the GBE CQM for vector mesons.

Aside from spectroscopy the semirelativistic GBE CQM has also been used to determine hadronic decay widths of baryons using perturbative calculations employing elementary emission [12, 13, 14] (without introducing additional parameters) and also pair creation (one additional parameter) models [15]. At that stage a satisfactory description of experimental data was impossible. A relativistic treatment of hadronic decays in an elementary-emission-type model seemed natural [16] and recently a Poincaré invariant decay model along the point-form spectator approximation has been suggested 17, 18]. It was observed that, in general, the theoretical results considerably underestimated the experimental data. In the light of these results the next natural step is to couple the decay channels explicitly to the $q q q$ (for baryons) and $\bar{q} q$ (for mesons) channels, respectively. In $[1,[19,20]$ a study of vector mesons has been done to investigate the effects of such a dynamical treatment of the exchange particle in the GBE in a Poincaré invariant framework both in terms of mass shifts and decay widths. The semirelativistic form of the model produces a much too large mass splitting of $\varrho$ and $\omega$. This flaw is removed in the $\mathrm{CC}$ treatment, leading to small mass shifts from the GBE in vector mesons, which confirmes the expectation that this type of interaction should not contribute much to the binding of such states. Regarding hadronic decays, the situation is difficult to judge with regard to comparison with experimental data, since for only one branching ratio, which can be calculated in the model [1], there is actually available data 21].

In the present work we follow Ref. 1], which contains the details of all aspects of the formalism and model used here, applying those to axial-vector mesons with quantum numbers $J^{P C}=1^{++}$and $J^{P C}=1^{+-}$as well as the strange sector via mixing of the states with the respective quantum numbers. The results identify dynamical effects for $l=1$ and enable the calculation of more hadronic decay widths within the restrictions of the states contained in the model. We briefly review the main ingredients of the model in Sec. III the results are presented and discussed in Sec. III and Sec. IV] contains conclusions.

\section{MODEL AND APPROXIMATIONS}

The central point of the CC treatment described in 1] is the CC mass operator, which is defined on the little Hilbert space of the direct sum of $\mathcal{H}_{\bar{q} q}$ (quark-antiquark)

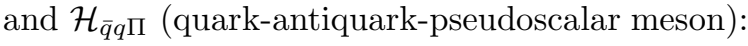

$$
M=M_{c}+M_{I}=\left(\begin{array}{cc}
\mathcal{D}_{\bar{q} q}^{c} & 0 \\
0 & \mathcal{D}_{\bar{q} q \Pi}^{c}
\end{array}\right)+\left(\begin{array}{cc}
0 & K^{\dagger} \\
K & 0
\end{array}\right)
$$


Here $M_{c}$ represents the diagonal part of $M$, which includes the confinement interaction such that in both the $\bar{q} q$ and $\bar{q} q \Pi$ channels the $\bar{q} q$ pair is confined. $M_{I}$ contains a vertex piece $K$ and its hermitian adjoint as defined in Eqs. (31) and (32) of [1]. When the eigenvalue equation for $M$ is reduced to the $\bar{q} q$ channel, one obtains the effective interaction term on the right-hand side of

$$
\left(\mathcal{D}_{\bar{q} q}^{c}-m\right)\left|\Psi_{\bar{q} q}\right\rangle=K^{\dagger}\left(\mathcal{D}_{\bar{q} q \Pi}^{c}-m\right)^{-1} K\left|\Psi_{\bar{q} q}\right\rangle .
$$

In this equation, $m$ is the eigenvalue and appears also in the effective interaction term. We note here that the interaction contains terms which correspond to the exchange of a pseudoscalar meson $\Pi$ inside the $\bar{q} q$ pair and others, in which the pseudoscalar meson $\Pi$ couples to the same constituent twice. We will refer to the latter as "loop terms". The particular form and choices for the operators in Eq. (2) and their matrix elements are described in detail in the appendix of [1]. Extending the model with the parameters established in the vectormeson sector to the axial vectors, the question for the need for readjustment of some of the parameters arises. Already for the vector-meson calculation, the parameters used in the exchange part of the interaction were taken without change from the semirelativistic GBE CQM in [9] and we have kept them the same here as well. This includes the definition and parameters of the quark-meson vertex form factors which determine the range of the $\Pi$ exchange. For the confinement piece, a harmonic oscillator (HO) model was used in $M^{2}$ for two reaons: first, the HO mass operator's eigenvalues and eigensolutions are analytically known, which facilitates the calculations and second, it mimicks the spectrum of a linear confinement potential for $M$. The actual form used in [1] is

$$
\mathcal{D}_{\bar{q} q}^{c} \rightarrow M_{n l}=\sqrt{8 a^{2}(2 n+l+3 / 2)+V_{0}+4 \bar{m}^{2}},
$$

where $\bar{m}^{2}$ is determined by the constituent quark masses, and $n$ and $l$ are the radial and orbital angular momentum quantum numbers, respectively. $a$ is the confinement strength and $V_{0}$ a constant used to fix the mass of the $\varrho$ ground state to its physical value. $a$ was adjusted such that the splitting of the $\varrho$ ground and first excited states was reproduced. It should be noted here that this is not the best possible choice for $\mathcal{D}_{\bar{q} q}^{c}$ in terms of an accurate fit to experimental data for higher excited states. However, the main emphasis of our studies still lies on the effects of a dynamical treatment of the one-particle exchange as compared to an instantaneous approximation (IA). Therefore $\mathcal{D}_{\bar{q} q}^{c}$ is sufficient for the present purpose. In principle one could choose any confinement operator, which is diagonal in the basis of Eq. (2), satisfies the (in our case point-form) Bakamjian-Thomas requirements [10], and whose solutions are known and can therefore be used to discretize the problem.

In an attempt to extend the basic HO piece of the model beyond the vector-meson sector one can use the concept of Regge trajectories 22]. This approach has already been used in [4] and recently in the context of relativistic Hamiltonian dynamics 23. If one uses such a trajectory, which contains the $\varrho$ ground state, to determine the parameters $a$ and $V_{0}$, one finds that the parameter set established for the vector mesons does not need to be changed. The parameters used in all calculations presented here are thus $a=312 \mathrm{MeV}$ and $V_{0}=-1.04115$ $\mathrm{GeV}$. This completes the summary of the model definitions.

In the calculations we make two approximations. First, we do not treat the loop contributions in the effective interaction explicitly. This is motivated by the assumption that their effects can be accounted for via a change in the constituent-quark mass. An explicit treatment of these terms is an extension of the model which will be incorporated in future studies. Second, some of the matrix elements occurring in Eq. (2) contain Wigner rotations, which come from the overlap of the various sets of basis states used in the computation of the effective interaction (see Eq. (A16) in 1]). We neglect these rotations, because, while the numerical effort to include them is considerable, their effects have been found to be small compared to boost effects in calculations of electromagnetic form factors of the nucleon 24].

\section{RESULTS AND DISCUSSION}

We have obtained results for the axial-vector states with quantum numbers $J^{P C}=1^{++}$and $J^{P C}=1^{+-}$by solving the eigenvalue equation for $M$ numerically. For quark-model mesons one has the relations $P=(-1)^{l+1}$ and $C=(-1)^{l+s}$, where $l$ is the orbital angular momentum and $s$ the total spin of the constituents, respectively; for $J^{P C}=1^{++}$this entails $s=1, l=1$; the physical states corresponding to this set of quantum numbers are the $f_{1}$ (with isospin $\left.I=0\right), a_{1}(I=1)$, and $K_{1 A}$ $(I=1 / 2)$. for $J^{P C}=1^{+-}$one gets $s=0, l=1$ with the associated particles $h_{1}(I=0), b_{1}(I=1)$, and $K_{1 B}$ $(I=1 / 2)$. Within the isospin 0 channels we assume ideal mixing between the octet and singlet $S U(3)$-flavor configurations, meaning that the $h_{1}$ as well as the $f_{1}$ spectra each contain both pure $\bar{n} n$ and $\bar{s} s$ states (in the usual notation, $n$ here denotes light quarks). In the strange sector, the physical states of the $K_{1}$ spectrum are mixtures of the $K_{1 A}$ and $K_{1 B}$, since they are not charge-parity eigenstates. In our treatment of this mixing we follow Blundell et al. [25]. The results are presented in Fig. 1 in six "columns" for each set of quantum numbers; the experimental values 21] are depicted in the first column (denoted by the particle names) by boxes indicating the experimental uncertainties; the second to sixth columns contain results for pure oscillator $(\mathrm{O})$, coupled channel calculation with vertex form factor (Cf), CC calculation with the form factor set $=1(\mathrm{C} 1)$, an instantaneous approximation with vertex form factor (If), and the IA calculation with the form factor set $=1$ (I1). These are the same categories as presented in Ref. [1]; also see this ref- 


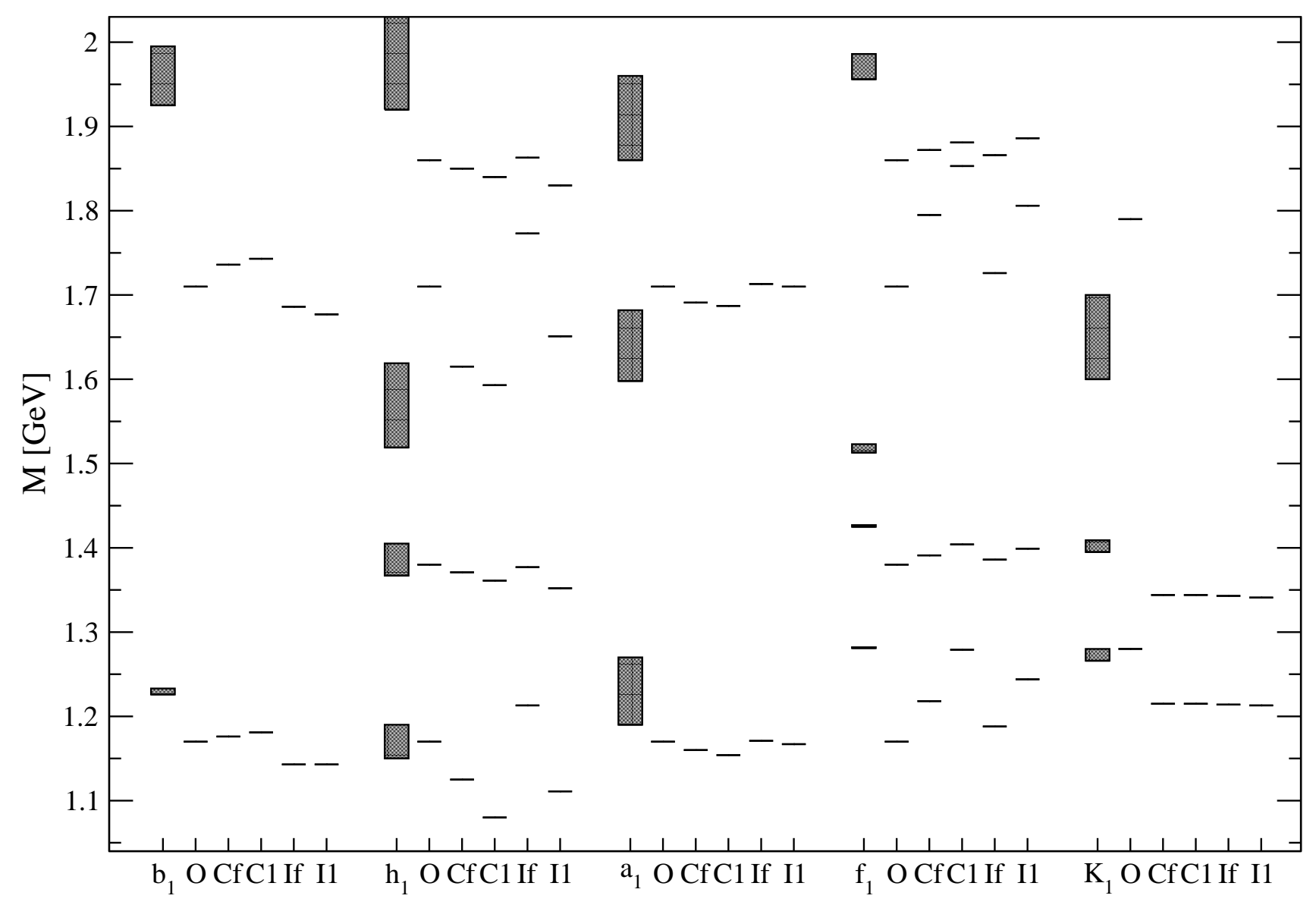

FIG. 1: The results for axial-vector meson spectra: Experimental data with uncertainties (particle name), oscillator (O), CC with vertex form factor $(\mathrm{Cf}), \mathrm{CC}$ with $\mathrm{FF}=1$ (C1), instantaneous approximation with vertex $\mathrm{FF}$ (If), and IA with $\mathrm{FF}=1$ (I1)

erence for details.

In the vector-meson sector [1] the $\bar{q} q$ states have mainly orbital angular momentum $l=0$ with small admixtures of $l=2$. There the main observation was that generally the CC treatment produces smaller mass shifts than the IA, including the prominent case of the $\omega$ ground state. For axial-vector mesons one always has $l=1$ and the observations about mass shifts are different, except that the main differences between CC treatment and IA can be found in the isoscalar channels; this is not surprising, because one-pion exchange is strongest in these channels and the light mass of the exchange particle plays a central role in the dynamical setup.

In general the dependence of the mass shifts on the use of a form factor at the quark-meson vertex is smaller in the CC treament than in the IA. This is true in particular for the case of the $h_{1}$ meson, where for the IA the shifts with and without the form factor have opposite sign. For the $b_{1}$, a different sign change appears: while the CC shifts are positive, the IA ones are negative (although in both cases the shifts are small). In the $f_{1}$ spectrum, one observes that mass shifts using the form factor are larger for the CC than the IA results and the same seems to apply to the results without the form factor except for the first $\bar{s} s$ state. This is opposite to the general observation for vector mesons. The reason for these variety of effects lies in the complexity of the dynamical setup for the $\mathrm{CC}$ formalism in connection with the wave functions for $l=1$ states. On different ranges of the relative momentum between the $q$ and the $\bar{q}$, these can have support of different sign, which gets modified in addition via the kinematical relations used in the calculation as apparent from Eq. (A16) in [1].

The results for the decay widths are larger than in the vector-meson case. However, similarly to the latter there is only one branching ratio in 21] which we can compare our results to, although most of the decays are regarded as "seen". Our results are generally of the order of $\approx 20$ to $90 \mathrm{MeV}$. The known branching ratio is that of the $b_{1}(1235)$ with $\Gamma=142 \pm 9 \mathrm{MeV}$, which decays dominantly into channels contained in our model; our result is $33 \mathrm{MeV}$ with and $42 \mathrm{MeV}$ without the form factor, underestimating experiment by about a factor of 4 . 


\section{CONCLUSIONS}

We have applied the Poincaré invariant coupledchannel (CC) formalism of Ref. 1] to the sector of axial-vector mesons. The axial-vector quantum numbers $J^{P C}=1^{++} / 1^{+-}$both imply orbital angular momentum $l=1$ for the $\bar{q} q$ pair. The effects of the dynamical treatment of the one-boson exchange (OBE) as compared to the instantaneous approximation (IA) reveals different characteristics as compared to the case of vector mesons (mainly $l=0$ ). There are four main observations: i) as for the vector mesons, the effects are strongest in the isoscalar channels, since there one-pion exchange dominates, which is very sensitive to the dynamical setup due to the light pion mass. ii) in the $h_{1}$ spectrum the use of a vertex form factor (as compared to a form factor $=1$ ) changes the sign of the mass shift from the meson exchange in the IA, while the $\mathrm{CC}$ results have the same sign and magnitude regardless of the details of the form factor. iii) in the $f_{1}$ spectrum the CC mass shifts are larger than the IA ones - opposite to the general trend (including the vector mesons). iv) in the $a_{1}$ spectrum the CC shifts have the opposite sign as compared to the IA ones. The main conclusion from this collection of observations must be that results from a dynamical CC treatment of one-particle exchange can differ significantly, both in magnitude and sign, in channels where this exchange is important. The results for the decay widths are sizable, but comparison with experimental data is inconclusive: the only data point with definite value (for the $b_{1}(1235)$ ) is underestimated by a factor of 4 . This supports the conclusion drawn in [1] from an analogous situation in the $\omega(1420)$ case: the calculations could be improved by explicitly including the loop contributions from the interaction in Eq. (2) and/or taking into account final-state interactions.

These conclusions strongly suggest analogous investigations of $q q q$ systems in this context, since such a dynamical, Poincaré invariant treatment of OBE in the baryon sector is yet missing. In [1] and the present work the path is laid out and also a possible intermediate step has been identified [26]. We note here that a treatment along the lines of the stochastic variational method 27] used up to date in the GBE CQM [9] seems impossible due to the high dimensions and numerical nature of the integrations involved in the solution of the CC problem. A more promising approach is of Faddeev type along the lines of Ref. 28]. It is important to proceed in this direction, because: a dynamical treatment of the OBE in baryons will yield hadronic baryon decay widths in a nonperturbative way, which could remedy their unsatisfactory description at the present stage of the model. Furthermore, given the limited comparison to experimental data of hadronic meson decays predicted by the present work and in Ref. 1] as well as the importance of GBE in the baryon sector, an analogous investigation of baryons will make the full impact of a dynamical treatment of OBE-type interactions clear.

\section{Acknowledgments}

We acknowledge valuable discussions with F. Coester, W. H. Klink, T. Melde, and W. Schweiger. This work was supported by: $F W F$ via Erwin-Schrödinger-Stipendium no. J2233-N08 and the Department of Energy, Office of Nuclear Physics, contract no. W-31-109-ENG-38.
[1] A. Krassnigg, W. Schweiger and W. H. Klink, Phys. Rev. C 67, 064003 (2003)

[2] S. Godfrey and N. Isgur, Phys. Rev. D 32, 189 (1985).

[3] S. Capstick and N. Isgur, Phys. Rev. D 34, 2809 (1986).

[4] R. P. Feynman, M. Kislinger and F. Ravndal, Phys. Rev. D 3, 2706 (1971).

[5] J. Carlson, J. B. Kogut and V. R. Pandharipande, Phys. Rev. D 27, 233 (1983).

[6] L. Y. Glozman and D. O. Riska, Phys. Rept. 268, 263 (1996)

[7] L. Y. Glozman, Z. Papp, W. Plessas, K. Varga and R. F. Wagenbrunn, Nucl. Phys. A 623, 90C (1997).

[8] L. Y. Glozman, Z. Papp and W. Plessas, Phys. Lett. B 381, 311 (1996)

[9] L. Y. Glozman, W. Plessas, K. Varga and R. F. Wagenbrunn, Phys. Rev. D 58, 094030 (1998)

[10] B. D. Keister and W. N. Polyzou, Adv. Nucl. Phys. 20, 225 (1991).

[11] T. Thonhauser, Master's thesis, Karl-Franzens-Universität Graz, 1998, unpublished.

[12] L. Y. Glozman, W. Plessas, L. Theussl, R. F. Wagenbrunn and K. Varga, PiN Newslett. 14, 99 (1998).

[13] A. Krassnigg, W. Plessas, L. Theussl, R. F. Wagenbrunn and K. Varga, Few Body Syst. Suppl. 10, 391 (1999).

[14] W. Plessas et al., Few Body Syst. Suppl. 11, 29 (1999).

[15] L. Theussl, R. F. Wagenbrunn, B. Desplanques and W. Plessas, Eur. Phys. J. A 12, 91 (2001)

[16] A. Krassnigg and W. Klink, Nucl. Phys. Proc. Suppl. 90, 25 (2000).

[17] T. Melde, R. F. Wagenbrunn and W. Plessas, Few Body Syst. Suppl. 14, 37 (2003)

[18] T. Melde, W. Plessas and R. F. Wagenbrunn, arXiv:hep-ph/0406023

[19] A. Krassnigg, W. Schweiger and W. H. Klink, Few Body Syst. Suppl. 14, 403 (2003).

[20] A. Krassnigg, W. Schweiger and W. H. Klink, Few Body Syst. Suppl. 14, 419 (2003).

[21] S. Eidelman et al. [PDG], Phys. Lett. B 592, 1 (2004).

[22] A. Tang and J. W. Norbury, Phys. Rev. D 62, 016006 (2000)

[23] V. V. Andreev and M. N. Sergeenko, arXiv:hep-ph/9912299

[24] R. F. Wagenbrunn, S. Boffi, W. Klink, W. Plessas and M. Radici, Phys. Lett. B 511, 33 (2001)

[25] H. G. Blundell, S. Godfrey and B. Phelps, Phys. Rev. D 53, $3712(1996)$ 
[26] W. Klink et al., Prepared for Mini-Workshop on Selected Few Body Problems in Hadronic and Atomic Physics, Bled, Slovenia, 7-14 Jul 2001

[27] K. Varga and Y. Suzuki, Phys. Rev. C 52, 2885 (1995)
[28] Z. Papp, A. Krassnigg and W. Plessas, Phys. Rev. C 62, 044004 (2000) 